\title{
INFLUÊNCIA DO ÂNGULO ENTRE O ESFORÇO APLICADO E A DIREÇÃO DAS FIBRAS DA MADEIRA SOBRE O MÓDULO DE ELASTICIDADE
}

\author{
Norman Barros Logsdon ${ }^{1}$, Zenésio Finger ${ }^{2}$, José Manoel Henriques de Jesus ${ }^{3}$ \\ ${ }^{1}$ Eng. Civil, Dr., Faculdade de Engenharia Florestal, UFMT, Cuiabá, MT, Brasil - logsdon@terra.com.br \\ ${ }^{2}$ Eng. Florestal, Dr., Faculdade de Engenharia Florestal, UFMT, Cuiabá, MT, Brasil - fingerz@ terra.com.br \\ ${ }^{3}$ Eng. Civil, Dr., Faculdade de Arquitetura, Engenharia e Tecnologia, UFMT, Cuiabá, MT, Brasil - jmhenriques@ terra.com.br
}

Recebido para publicação: 20/10/2009 - Aceito para publicação: 07/04/2010

\begin{abstract}
Resumo
A norma brasileira NBR 7190 - Projeto de Estruturas de Madeira - prevê a correção da resistência às tensões normais quando o ângulo entre o esforço e a direção das fibras $(\alpha)$ for maior que $6^{\circ} \mathrm{e}$ recomenda aplicar a expressão de Hankinson aos valores de cálculo, mas é omissa sobre a correção do módulo de elasticidade. Este trabalho objetivou levantar na literatura outras expressões para representar a variação do módulo de elasticidade com o ângulo $\alpha$ e verificar a validade destas expressões em valores médios. Foi utilizada uma amostra de doze pranchas de madeira, de cada prancha foram retirados corpos-de-prova de compressão, com diferentes inclinações das fibras, que foram ensaiados segundo a atual NBR 7190, fornecendo os módulos de elasticidade. Dos resultados de uma prancha obteve-se a variação dos módulos de elasticidade com o ângulo $\alpha$. Fixada uma inclinação obteve-se, em acordo com a NBR 7190, a rigidez efetiva (módulo de elasticidade médio) para a inclinação fixada. Destes valores médios obteve-se a variação da rigidez efetiva com o ângulo $\alpha$. Quatro modelos (Hankinson, Senos, Karlsen e Keylwerth) foram avaliados, utilizando-se os testes de "pairing" e dos desvios, e o que melhor representou o fenômeno, nos dois casos, foi o de Keylwerth.

Palavras-chave: Módulo de elasticidade; rigidez efetiva; inclinação das fibras.
\end{abstract}

\begin{abstract}
Influence of the angle between applied stress and wood grain direction on the module of elasticity. The Brazilian Code, NBR 7190 - Design of Timber Structures, foresees the correction of the strength to the normal tensions when the angle between the effort and the direction of the fibers $(\alpha)$ is larger than $6^{\circ}$ and recommends the application of Hankinson's expression to the calculated values, but is remiss about the module of elasticity correction. This work aimed to search in the literature other expressions to represent the variation of the module of elasticity with the angle and to verify the validity of these expressions in mean values. A sample comprised of 12 boards was used. From each board compression test blocks with different grain inclinations were taken and tested according the NBR 7190, providing the modules of elasticity for different angles. From the results of one board the module of elasticity variation, with the $\alpha$ angle, was obtained. After setting an inclination it was possible to obtain (according the NBR 7190) the effective stiffness (average module of elasticity) for the fixed inclination. Based on these mean values the variation of the effective stiffness with the $\alpha$ angle was obtained. Four models (Hankinson, Sins, Karlsen and Keylwerth) were evaluated, using the pairing and the residues tests. In both cases the Keylwerth model was the most efficient to represent the phenomenon.
\end{abstract}

Keywords: Module of elasticity; effective stiffness; fibers inclination.

\section{INTRODUÇÃO}

A atual norma brasileira "NBR 7190 - Projeto de Estruturas de Madeira", da Associação Brasileira de Normas Técnicas - ABNT (1997), prevê a correção da resistência de cálculo, para verificação de tensões normais inclinadas, sempre que o ângulo entre o esforço e a direção das fibras for 
superior a $6^{\circ}$, e recomenda, para essa correção, a aplicação da expressão de Hankinson. Já para correção da rigidez, necessária à avaliação de deformações ou deslocamentos, a atual norma brasileira não prevê correção.

Hankinson, segundo Hellmeister (1983), estudou a variação da tensão de ruptura com o ângulo entre a direção das fibras e o esforço, todavia alguns autores recomendam o uso da expressão de Hankinson também para o módulo de elasticidade.

A rigidez efetiva, segundo a NBR 7190 da ABNT (1997), é obtida a partir do valor médio do módulo de elasticidade, ao qual é aplicado um coeficiente de modificação. Não se encontrou, na bibliografia pesquisada, estudos relacionando rigidezes efetivas com o ângulo entre o esforço e a direção das fibras.

Essas informações preliminares indicam que o estudo da variação do módulo de elasticidade com o ângulo entre o esforço e a direção das fibras ainda não está concluído, pois a atual norma brasileira (NBR 7190,1997) é omissa a respeito e não se tem garantia experimental de que uma relação estudada para valores do módulo de elasticidade também possa ser aplicada às rigidezes efetivas, embora alguns autores tenham admitido, implicitamente, a validade dessa relação também para valores médios.

Partindo da hipótese de existir relação funcional entre o módulo de elasticidade e o ângulo entre o esforço aplicado e a direção das fibras da madeira, o objetivo deste trabalho foi estudar, teórica e experimentalmente, a influência do ângulo entre o esforço aplicado e a direção das fibras sobre o módulo de elasticidade da madeira e, especialmente, sobre sua rigidez efetiva.

\section{REVISÃO BIBLIOGRÁFICA}

Devido à natureza de sua fisiologia, a madeira, segundo Dinwoodie (1981), é um material higroscópico e anisotrópico. Por ser um material higroscópico, suas propriedades são muito influenciadas pela variação de seu teor de umidade, e, por ser um material anisotrópico, suas propriedades mecânicas dependem da direção de aplicação do esforço em relação às suas fibras.

A influência do ângulo entre o esforço aplicado e a direção das fibras fica evidente ao se estudarem as propriedades de tração paralela às fibras, que é a maior resistência apresentada pela madeira, e de tração normal às fibras, que é a menor resistência apresentada pela madeira.

Vários autores estudaram o assunto, alguns estabelecendo modelos teóricos, em geral considerando a madeira como um material ortotrópico, outros estabelecendo modelos empíricos, baseados em resultados de ensaios. A maioria desses modelos foi idealizada para estudar a variação da resistência (tensão de ruptura) com a direção das fibras, mas pode ser utilizado também para o módulo de elasticidade.

Entre os modelos empíricos, segundo Kollmann; Côté Jr. (1984), o modelo proposto por Hankinson, apresentado na equação 1 , com expoente $n=2$, tem sido o mais utilizado para se obter o módulo de elasticidade em uma direção inclinada. Karlsen et al. (1967), por outro lado, recomendam, para resistências, o modelo empírico apresentado na equação 2 , com expoente $\mathrm{n}=3$.

Um estudo teórico, desenvolvido por Szücs (1992), concluiu sobre a possibilidade de se utilizar para a correção de resistências, às tensões normais inclinadas, tanto por tração como por compressão, a equação 3, com o expoente $n=2$. Já segundo Pfeil (1978), a norma alemã DIN-1052 adota essa expressão com o expoente $\mathrm{n}=1$. Keylwerth, segundo Kollmann; Côté Jr. (1984), também desenvolveu uma expressão teórica, de expoente $\mathrm{n}=2$, para a variação do módulo de elasticidade, apresentada na equação 4.

Apenas o modelo de Keylwerth (equação 4) foi idealizado especificamente para o módulo de elasticidade. O modelo de Hankinson (equação 1), embora idealizado para resistências, também tem sido utilizado para o módulo de elasticidade. Os demais modelos fora idealizados para resistências, mas neste trabalho serão avaliados para o módulo de elasticidade.

$$
\begin{aligned}
& \mathrm{E}_{\alpha}=\frac{\mathrm{E}_{0} \cdot \mathrm{E}_{90}}{\mathrm{E}_{0} \cdot \operatorname{sen}^{\mathrm{n}} \alpha+\mathrm{E}_{90} \cdot \cos ^{\mathrm{n}} \alpha} \\
& \mathrm{E}_{\alpha}=\frac{\mathrm{E}_{0}}{1+\left(\frac{\mathrm{E}_{0}}{\mathrm{E}_{90}}-1\right) \cdot \operatorname{sen}^{\mathrm{n}} \alpha}
\end{aligned}
$$




$$
\begin{aligned}
& \mathrm{E}_{\alpha}=\mathrm{E}_{0}-\left(\mathrm{E}_{0}-\mathrm{E}_{90}\right) \cdot \operatorname{sen}^{\mathrm{n}} \alpha \\
& \mathrm{E}_{\alpha}=\frac{\mathrm{E}_{0}}{\left(\cos ^{\mathrm{n}} \alpha-\frac{\mathrm{E}_{0}}{\mathrm{E}_{90}} \operatorname{sen}^{\mathrm{n}} \alpha\right) \cdot \cos (2 \cdot \alpha)+\frac{\mathrm{E}_{0}}{\mathrm{E}_{45}} \operatorname{sen}^{\mathrm{n}}(2 \cdot \alpha)}
\end{aligned}
$$

Em que:

$\mathrm{E}_{\alpha}=$ módulo de elasticidade na direção inclinada de um ângulo $\alpha$ em relação às fibras da madeira;

$\mathrm{E}_{0}=$ módulo de elasticidade na direção paralela $\left(\alpha=0^{\circ}\right)$ em relação às fibras da madeira;

$\mathrm{E}_{45}=$ módulo de elasticidade na direção inclinada de um ângulo $\alpha=45^{\circ}$ em relação às fibras da madeira;

$\mathrm{E}_{90}=$ módulo de elasticidade na direção perpendicular $\left(\alpha=90^{\circ}\right)$ em relação às fibras da madeira;

$\alpha=$ ângulo entre o esforço aplicado e a direção das fibras;

$\mathrm{n} \quad=$ expoente dos termos trigonométricos.

Também há controvérsias, entre os autores, sobre o valor de expoente dos termos trigonométricos (n) nas equações 1 a 4. Alguns autores indicam coeficientes diferentes conforme o tipo de solicitação em análise, enquanto outros indicam coeficientes diferentes para diferentes teores de umidade da madeira.

Tomando como exemplo a expressão de Hankinson (equação 1) encontrou-se, na literatura, as seguintes informações: o expoente n, segundo Szücs (1992), pode variar de 1,5 a 3, conforme a análise que está sendo realizada; a atual norma brasileira, NBR 7190 da ABNT (1997), adota o expoente $n=2$ para a correção de resistências de cálculo, às tensões normais inclinadas, tanto por tração como por compressão; Kollmann; Côté Jr. (1984) mostram que valores do expoente n entre 1,5 e 2,0 são satisfatórios para os esforços de tração, mas para esforços de compressão em madeira seca dever-se-ia utilizar $n=2,5$; Mateus (1962) recomenda a aplicação da expressão de Hankinson, com o expoente $n=2$ para a correção de resistências às tensões normais inclinadas, tanto por tração como por compressão; já Nicolas; Mascia; Trinca (2006) concluíram ser adequado utilizar 1,5 $\leq n \leq 2,0$ para a tração.

Percebe-se, pela revisão bibliográfica realizada, que o estudo da variação do módulo de elasticidade com o ângulo entre o esforço aplicado e a direção das fibras ainda não está concluído. Não existe consenso entre autores sobre qual a melhor expressão a ser utilizada e nem mesmo sobre o valor do expoente em determinada expressão.

Todos os estudos obtidos na pesquisa bibliográfica apontam para a variação em um indivíduo (ou para a média dos resultados obtidos em diferentes indivíduos), mas a aplicação em projetos de estruturas de madeira utiliza-se de valores efetivos de rigidez, que se originam de valores médios. Valores médios, além da variação individual do efeito em questão, estão associados à variabilidade de resultados dentro de uma árvore e ao longo das árvores da espécie do lote avaliado. Dessa forma, torna-se necessário avaliar como variam valores efetivos de rigidez quando se varia o ângulo entre o esforço aplicado e a direção das fibras.

\section{MATERIAL E MÉTODOS}

\section{Amostragem}

Para estudar a variação do módulo de elasticidade com o ângulo entre o esforço aplicado e a direção das fibras, é necessário utilizar-se material com a menor variação possível das características gerais entre suas amostras, pois, ao se utilizar uma amostragem heterogênea, segundo Logsdon (1998), pode-se cometer um erro na especificação do modelo adotado.

Dada a impossibilidade de se utilizar o mesmo corpo de prova, tendo em vista não se poder alterar a direção das fibras do corpo de prova, podem-se utilizar corpos de prova semelhantes (de material o mais homogêneo possível). Logsdon (1995), estudando a variação da resistência ao cisalhamento paralelo às fibras ao longo de uma peça de dimensões comerciais de amescla, Trattinikia burserifolia 
(Mart.) Wild., concluiu que, ao longo do comprimento, essa variação é tão pequena que pode ser desprezada. Assim, corpos de prova semelhantes podem ser obtidos de uma mesma barra ou prancha.

A rigidez efetiva, segundo a NBR 7190 da ABNT (1997), é obtida a partir do valor médio do módulo de elasticidade, que, por sua vez, é obtido do ensaio de compressão.

Para estudar a variação da rigidez efetiva com o ângulo entre o esforço aplicado e a direção das fibras, por sua vez, é necessário considerar a variabilidade da madeira dentro de uma árvore e entre as árvores da espécie.

A atual norma brasileira, NBR 7190 da ABNT (1997), define como amostragem para um lote homogêneo de até $12 \mathrm{~m}^{3}$ de madeira uma amostra de 12 corpos de prova para cada tipo de ensaio.

Assim, tomou-se um lote mínimo formado por três árvores. De cada árvore foram retiradas quatro pranchas diametrais (plano radial-longitudinal), totalizando 12 pranchas. De cada prancha foram retirados os corpos de prova como se apresenta na figura 1. Dessa forma, cada inclinação contou com 12 corpos de prova, totalizando 132 corpos de prova de compressão $\left(\mathrm{a} 0^{\circ}, 10^{\circ}, 20^{\circ}, 30^{\circ}, 40^{\circ}, 45^{\circ}, 50^{\circ}, 60^{\circ}\right.$, $70^{\circ}, 80^{\circ}$ e $90^{\circ}$ ). O material não utilizado foi identificado e armazenado de modo a permitir refazer qualquer corpo de prova cujo resultado de ensaio apresentasse anomalia.

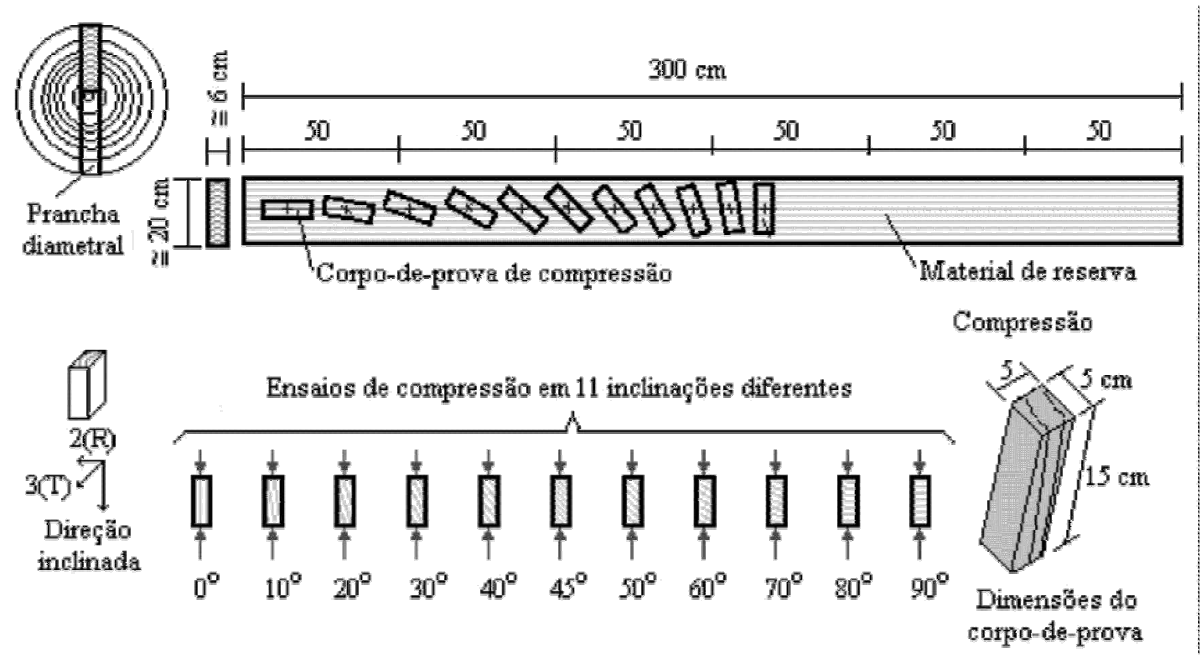

Figura 1. Plano de amostragem dos corpos de prova de uma prancha.

Figure 1. Sampling method to obtain the specimens from a board.

\section{Espécie utilizada}

Devido à facilidade de obtenção, utilizou-se a espécie Aspidosperma populifolium A. DC., conhecida por peroba-mica, uma vez que é uma das espécies em exploração no extremo norte do estado de Mato Grosso. O material foi coletado no município de Cotriguaçu-MT, em área de floresta amazônica explorada pela empresa MR Madeiras. As árvores tinham pelo menos 100 anos e diâmetro superior a $100 \mathrm{~cm}$. Essa espécie, segundo Logsdon et al. (2008), possui moderada densidade aparente $\left(\rho_{\text {ap, } 12 \%} \cong 726 \mathrm{~kg} / \mathrm{m}^{3}\right)$ e elevada resistência mecânica, considerando que sua resistência característica à compressão paralela às fibras $\left(f_{c 0, k} \cong 64,98 \mathrm{MPa}\right)$ a posiciona na classe de resistência $\mathrm{C} 60$, a mais resistente entre as classes definidas pela ABNT (1997) para as dicotiledôneas.

\section{Secagem prévia}

A fim de estabilizar a madeira a um teor de umidade próximo de $12 \%$, de modo a minimizar erros ao reportar os resultados dos ensaios ao teor de umidade de referência, foi providenciada uma secagem ao ar da madeira.

Inicialmente a madeira foi desdobrada em peças, ligeiramente maiores que os corpos de prova, com seção de aproximadamente $6 \mathrm{~cm} \times 6 \mathrm{~cm}$, conforme o esquema da figura 1. As peças foram identificadas e colocadas para secar ao ar até que a massa das peças de uma amostra de controle, composta por três peças (uma de cada árvore), apresentasse, simultaneamente, constância de massa 
(variação inferior a $0,5 \%$ ) entre duas leituras sucessivas, no intervalo de um dia. Essa secagem prévia teve uma duração aproximada de 30 dias.

Só após essa secagem prévia as peças foram desdobradas em corpos de prova. Esse procedimento minimizou a ocorrência de deformações dos corpos de prova, por variação do teor de umidade.

\section{Ensaios de compressão}

Os ensaios de compressão, dos quais foram obtidos os módulos de elasticidade, foram realizados em corpos de prova de dimensões $5 \mathrm{~cm} \times 5 \mathrm{~cm} \times 15 \mathrm{~cm}$, com as direções das fibras bem definidas, utilizando-se a metodologia descrita na NBR 7190 da ABNT (1997), para o ensaio de compressão paralela às fibras. A massa de cada corpo de prova foi avaliada logo após o ensaio e após a secagem em estufa, a fim de se obter a umidade no instante do ensaio, possibilitando reportar o resultado ao teor de umidade de referência de $12 \%$.

Os ensaios foram realizados com dois ciclos iniciais de carga e um final. Os ciclos iniciais se limitaram a $50 \%$ da estimativa da tensão de ruptura. No ciclo final (terceiro), o carregamento foi aumentado gradativamente até a ruptura.

A estimativa da tensão de ruptura para cada ensaio (diferentes inclinações) da mesma prancha foi obtida a partir do ensaio de compressão paralela, realizado em outro corpo de prova (um para cada prancha). Esse corpo de prova forneceu uma estimativa da tensão de ruptura à compressão paralela às fibras $\left(\mathrm{f}_{\mathrm{c} 0}\right)$. A estimativa da tensão de ruptura à compressão normal às fibras $\left(\mathrm{f}_{\mathrm{c} 90}\right)$ foi obtida pela aplicação da equação 5, fornecida pela NBR 7190 da ABNT (1997), para caracterização simplificada de madeiras. Finalmente, para se obter a estimativa da tensão de ruptura à compressão inclinada de um ângulo " $\alpha$ " em relação às fibras $\left(f_{c \alpha}\right)$, aplicou-se a expressão de Hankinson, com expoente " $n=2$ ", apresentada na equação 6 .

$$
\begin{aligned}
& \frac{\mathrm{f}_{\mathrm{c} 90}}{\mathrm{f}_{\mathrm{c} 0}}=0,25 \Rightarrow \mathrm{f}_{\mathrm{c} 90}=0,25 \cdot \mathrm{f}_{\mathrm{c} 0} \\
& \mathrm{f}_{\mathrm{c} \alpha}=\frac{\mathrm{f}_{\mathrm{c} 0} \cdot \mathrm{f}_{\mathrm{c} 90}}{\mathrm{f}_{\mathrm{c} 0} \cdot \operatorname{sen}^{2} \alpha+\mathrm{f}_{\mathrm{c} 90} \cdot \cos ^{2} \alpha}
\end{aligned}
$$

No início e fim de cada ciclo, a carga foi mantida constante durante 30 segundos, como recomenda a NBR 7190 da ABNT (1997). Desses ensaios, no início/fim de cada ciclo e a cada 10\% da tensão de ruptura estimada, no ciclo final, foram anotadas a carga e a deformação. Também foram anotadas a área da seção transversal do corpo de prova e a massa do corpo de prova, no fim do ensaio e após completa secagem.

Assim, com os dados registrados no ciclo final (terceiro), foi possível obter o módulo de elasticidade (equação 7) e o teor de umidade dos corpos de prova no instante do ensaio (equação 8).

$$
\begin{aligned}
& \mathrm{E}_{\alpha}=\frac{\sigma_{50 \%}-\sigma_{10 \%}}{\varepsilon_{50 \%}-\varepsilon_{10 \%}} \\
& \mathrm{U}=\frac{\mathrm{m}_{\mathrm{u}}-\mathrm{m}_{0}}{\mathrm{~m}_{0}} .100 \%
\end{aligned}
$$

Em que:

$$
\begin{array}{ll}
\mathrm{E}_{\alpha} & =\begin{array}{l}
\text { módulo de elasticidade na direção inclinada de um ângulo } \alpha \text { em relação às } \\
\text { fibras da madeira; }
\end{array} \\
\sigma_{10 \%} \text { e } \sigma_{50 \%} & =\begin{array}{l}
\text { tensões de compressão correspondentes a } 10 \% \text { e } 50 \% \text { da resistência estimada } \\
\mathrm{f}_{\text {c⿱ }} \text {, em } \mathrm{MPa} ;
\end{array} \\
\varepsilon_{10 \%} \text { e } \varepsilon_{50 \%} & =\begin{array}{l}
\text { deformações específicas medidas no corpo de prova, correspondentes às } \\
\text { tensões de } \sigma_{10 \%} \text { e } \sigma_{50 \%} ;
\end{array} \\
\mathrm{U} & =\text { teor de umidade do corpo de prova no instante do ensaio, em } \% ; \\
\mathrm{m}_{\mathrm{u}} & =\text { massa do corpo de prova no instante do ensaio, em g; } \\
\mathrm{m}_{0} & =\text { massa do corpo de prova seco em estufa, em g. }
\end{array}
$$


Para avaliação de massas foi utilizada uma balança analítica, com capacidade de $1,0 \mathrm{~kg}$ e sensibilidade de $0,01 \mathrm{~g}$. As dimensões foram avaliadas com um paquímetro digital, com capacidade de $10 \mathrm{~cm}$ e sensibilidade de $0,01 \mathrm{~mm}$. A secagem foi feita em estufa de esterilização e secagem que mantinha temperatura de $(103 \pm 2)$ oC. A aplicação da carga foi feita em máquina universal de ensaios, com capacidade de $300 \mathrm{kN}$ e sensibilidade de $0,01 \mathrm{kN}$, a uma velocidade de carregamento de $10 \mathrm{MPa} / \mathrm{min}$, e as deformações foram obtidas por meio de extensômetros eletrônicos com sensibilidade de $0,5 \mu \mathrm{m}$.

Para reportar os resultados ao teor de umidade de referência, foi utilizada a equação aferida por Logsdon (1998), apresentada na equação 9, já com a notação adequada ao caso.

$$
\mathrm{E}_{12}=\mathrm{E}_{\mathrm{U} \%} \cdot\left[1+\frac{2,5 \cdot(\mathrm{U} \%-12)}{100}\right]
$$

Em que:

$\mathrm{E}_{12}=$ módulo de elasticidade, obtido no ensaio de compressão, ao teor de umidade de referência de 12\%;

$\mathrm{E}_{\mathrm{U} \%}=$ módulo de elasticidade, obtido no ensaio de compressão, ao teor de umidade $\mathrm{U} \%$;

$\mathrm{U} \%=$ teor de umidade da madeira no instante do ensaio, em \%.

\section{Cuidados iniciais}

Todos os modelos apresentados na revisão bibliográfica para representar a variação do módulo de elasticidade com o ângulo entre o esforço aplicado e a direção das fibras são dependentes de pelo menos dois valores experimentais, o módulo de elasticidade na direção paralela às fibras $\left(\alpha=0^{\circ}\right)$ e na direção normal às fibras $\left(\alpha=90^{\circ}\right)$. Assim, uma eventual falha na avaliação desses valores, por má condução do ensaio ou pela existência de algum defeito localizado na madeira, que não foi percebido, comprometeria a avaliação do modelo. Para minimizar erros na avaliação dos modelos, decidiu-se refazer todo ensaio cujo resultado destoasse de seus pares.

\section{Valores médios}

Dos 12 corpos de prova com a mesma direção das fibras foram obtidos os valores médios (equação 10) e o intervalo de confiança da média (equação 11).

$$
\begin{aligned}
& x_{w m}=\frac{\sum_{i=1}^{n} x_{i}}{n} \\
& \bar{x}-t(\phi, 95 \%) \cdot \frac{s_{x}}{\sqrt{n}} \leq \mu_{x} \leq \bar{x}+t(\phi, 95 \%) \cdot \frac{s_{x}}{\sqrt{n}}
\end{aligned}
$$

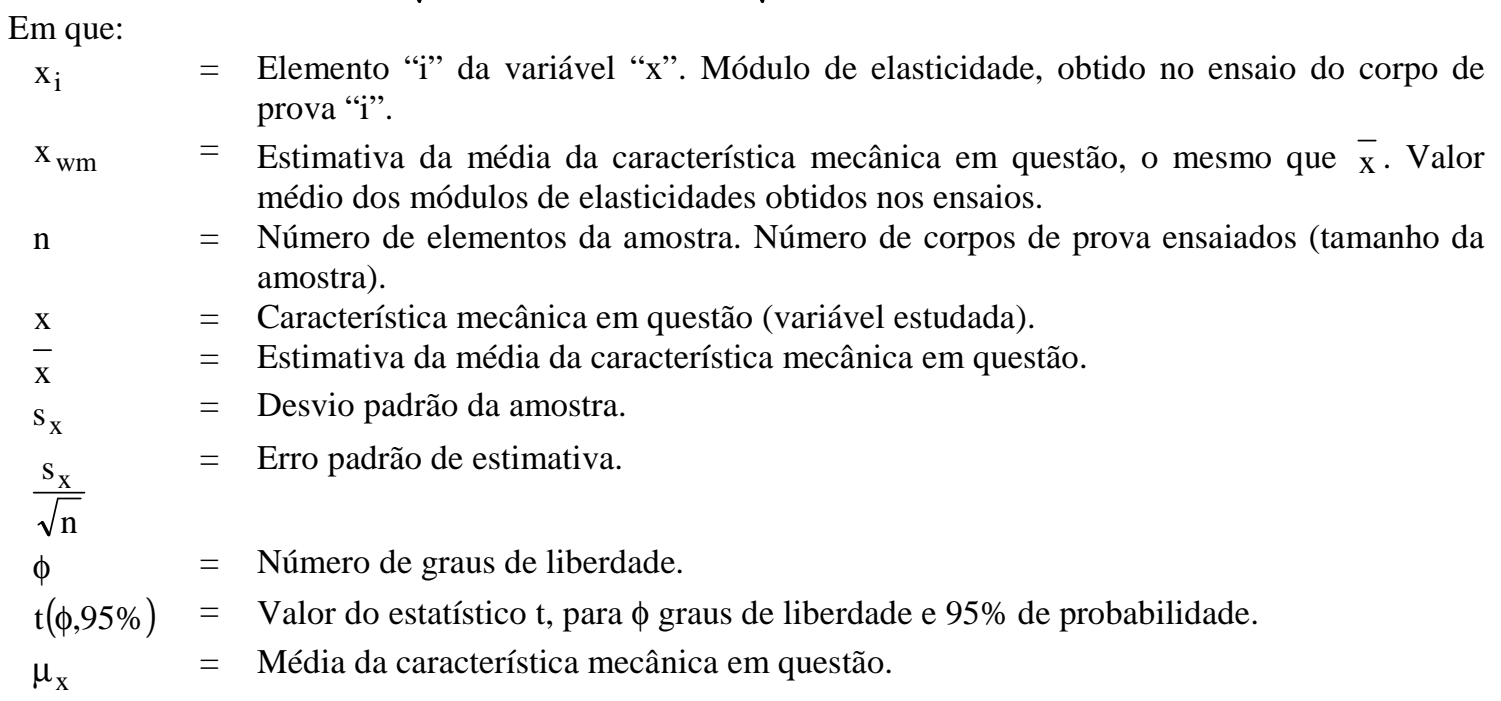




\section{Análise estatística}

Para avaliação dos modelos, foram utilizados dois instrumentos estatísticos: o teste de pairing, apresentado por Lahr (1983), que avalia se os desvios entre os resultados de ensaio e os obtidos pelo modelo têm média nula, e o teste dos desvios, apresentado por Logsdon (1998), que avalia se esses desvios não apresentam tendenciosidade ao longo do domínio.

Embora verossímil e tenha sido admitida implicitamente por alguns autores, não se encontrou nenhuma constatação estatística que permita afirmar que a "curva das médias" de um comportamento seja a "curva do comportamento médio".

Para avaliar a variação do módulo de elasticidade com o ângulo entre o esforço aplicado e a direção das fibras da madeira, optou-se por estudar o comportamento dos resultados obtidos em cada prancha, já corrigidos para o teor de umidade de referência de $12 \%$, e, em seguida, verificar a existência de um comportamento comum às 12 pranchas.

Para avaliar a variação da rigidez efetiva com o ângulo entre o esforço aplicado e a direção das fibras, optou-se por estudar o comportamento do valor médio do módulo de elasticidade.

Foram utilizados os modelos apresentados nas equações 1 a 4.

Para a análise estatística de cada prancha (ou do módulo de elasticidade médio), preparou-se uma planilha eletrônica, no aplicativo Microsoft Excel, na qual os resultados foram pareados aos valores calculados com os modelos, em que o expoente " $n$ " podia ser alterado. Desses pares de valores foram obtidos os desvios $\left(\mathrm{d}=\mathrm{E}_{\text {experimental }}-\mathrm{E}_{\text {modelo }}\right)$, o correspondente intervalo de confiança da média (equação 11) e o diagrama dos desvios $(\mathrm{dx} \alpha)$. O valor do expoente "n" foi sistematicamente alterado (com incrementos de 0,01), até se obter o intervalo em que o valor de "n" atendesse, simultaneamente, o teste de pairing (intervalo de confiança da média contendo o zero) e o teste dos desvios (desvios não apresentando tendenciosidade ao longo do domínio).

Os modelos que apresentaram um intervalo de " $\mathrm{n}$ " aceitável em todas as pranchas podem ser utilizados para representar a variação do módulo de elasticidade com o ângulo entre o esforço aplicado e a direção das fibras da madeira, com qualquer valor de " $n$ " do referido intervalo. Nessa avaliação, o erro estatístico pode ser minimizado retirando-se os valores destoantes de "n" (out limits).

\section{RESULTADOS E DISCUSSÃO}

Como não foi observado nenhum resultado destoante nos ensaios de compressão, não houve necessidade de refazer nenhum ensaio.

Os ensaios de compressão forneceram os valores dos módulos de elasticidades inclinados, já corrigidos para o teor de umidade de $12 \%$, apresentados na tabela 1 .

Aplicando o teste de pairing e o teste dos desvios, na comparação dos resultados dos ensaios (Tabela 1) aos dos modelos apresentados nas equações 1 a 4, foram obtidos, para cada prancha, os limites do intervalo do expoente "n" com validade estatística, bem como o valor do expoente "n" que produz o melhor ajuste de cada modelo.

Ao se tentar obter um intervalo de validade comum, de cada modelo, nas várias pranchas, foram obtidos alguns intervalos nos quais o limite inferior é maior que o limite superior ou em que o intervalo de validade não continha o melhor valor do expoente "n" para o conjunto, que seria o valor médio. Essas ocorrências foram classificadas como resultados destoantes (out limits).

Retirando-se os resultados destoantes (out limits), no máximo os dois resultados maiores e os dois menores, obtiveram-se resultados que indicam, para a madeira de peroba-mica, Aspidosperma populifolium A. DC.:

- A validade da Expressão de Hankinson (equação 1), desde que o expoente "n" esteja no intervalo $1,42 \leq n \leq 1,80$. O expoente que fornece melhor ajuste é $n=1,75$. O valor tradicionalmente utilizado, inclusive pela NBR 7190 da $A B N T$ (1997), de $n=2$, não tem validade estatística.

- A validade da Expressão dos Senos (equação 3), desde que o expoente " $n$ " esteja no intervalo $0,26 \leq n \leq 0,45$. O expoente que fornece melhor ajuste é $n=0,41$. O valor tradicionalmente utilizado pela norma alemã DIN-1052, segundo Pfeil (1978), de $n=1$, não tem validade estatística. Esse modelo também não tem validade com n $=2$, da proposta de Szücs (1992). 
- A validade da Expressão apresentada por Karlsen (equação 2), desde que o expoente "n" esteja no intervalo $1,44 \leq n \leq 1,74$. O expoente que fornece melhor ajuste é $n=1,65$. O valor tradicionalmente utilizado, recomendado por Karlsen et al. (1967), de $\mathrm{n}=3$, não tem validade estatística.

- A Expressão de Keylwerth (equação 4) tem validade estatística para "n" no intervalo 1,60 $\leq n \leq 2,90$. O expoente que fornece melhor ajuste é $n=2,01$. O valor tradicionalmente utilizado, recomendado por Kollmann; Côté Jr. (1984), de $\mathrm{n}=2$, também tem validade estatística. Esse foi o único modelo que apresentou validade com o expoente com o qual foi originalmente apresentado.

Tabela 1. Módulos de elasticidades obtidos nos ensaios de compressão de cada prancha.

Table 1. Modules of elasticity obtained in the compression tests of each board.

\begin{tabular}{|c|c|c|c|c|c|c|c|c|c|c|c|}
\hline \multirow{2}{*}{ 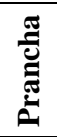 } & \multicolumn{11}{|c|}{$\begin{array}{l}\text { Valores dos módulos de elasticidades obtidos nos ensaios de compressão inclinada, } \\
\qquad \mathrm{E}_{\alpha}(\mathrm{MPa}) \text {, nas seguintes inclinações } \alpha \text { : }\end{array}$} \\
\hline & $\mathbf{0}^{\mathbf{0}}$ & $10^{\circ}$ & $20^{\circ}$ & $\mathbf{3 0}^{\mathbf{0}}$ & $40^{\circ}$ & $45^{\circ}$ & $\mathbf{5 0}^{\circ}$ & $60^{\circ}$ & $\mathbf{7 0}^{\circ}$ & $80^{\circ}$ & $90^{\circ}$ \\
\hline A1 & 12499 & 8335 & 4508 & 3463 & 2676 & 2443 & 2383 & 2045 & 1938 & 1808 & 1718 \\
\hline A2 & 15600 & 13831 & 8208 & 3802 & 2801 & 2482 & 2080 & 1858 & 1512 & 1437 & 1366 \\
\hline B1 & 10164 & 9436 & 5945 & 4040 & 2790 & 2739 & 2697 & 1879 & 2042 & 1913 & 1839 \\
\hline B2 & 15452 & 14188 & 7438 & 4573 & 3131 & 2869 & 2504 & 2825 & 2056 & 1512 & 1768 \\
\hline $\mathrm{C} 1$ & 14945 & 13736 & 7097 & 4136 & 3081 & 2895 & 2562 & 2009 & 1446 & 1912 & 1757 \\
\hline $\mathrm{C} 2$ & 13418 & 10859 & 7563 & 4611 & 3213 & 3043 & 2618 & 2101 & 1905 & 2073 & 1972 \\
\hline D1 & 13764 & 11650 & 6448 & 4300 & 3194 & 2890 & 2517 & 2279 & 2204 & 2054 & 2102 \\
\hline D2 & 11951 & 9393 & 6249 & 4061 & 3876 & 2976 & 2589 & 2291 & 2136 & 2112 & 2009 \\
\hline E1 & 16123 & 11615 & 5346 & 4396 & 2759 & 2338 & 2207 & 1721 & 1595 & 1533 & 1422 \\
\hline E2 & 10681 & 8752 & 5012 & 4481 & 3079 & 2776 & 2685 & 2224 & 2055 & 2118 & 1982 \\
\hline $\mathrm{F} 1$ & 14261 & 9812 & 6966 & 4353 & 2987 & 2524 & 2781 & 2248 & 2166 & 1806 & 2250 \\
\hline $\mathrm{F} 2$ & 14581 & 10788 & 5094 & 4072 & 3146 & 2793 & 2444 & 2107 & 2046 & 2045 & 2077 \\
\hline
\end{tabular}

Considerando que a Expressão de Keylwerth apresentou validade estatística no formato original, para $\mathrm{n}=2$; apresentou maior amplitude para o intervalo de validade, o que permite admitir a possibilidade de atender um número maior de espécies; tem origem em modelo teórico; utiliza um terceiro ponto experimental, que "força" o modelo a ajustar-se também para um ângulo de $45^{\circ}$; e pode ser escrita usando relações entre resistências, semelhantes às definidas pela atual NBR 7190 da ABNT (1997), resolveu-se avaliar o modelo baseado na Expressão de Keylwerth, apresentado na equação 12.

$$
\mathrm{E}_{\alpha}=\frac{\mathrm{E}_{0}}{\left(\cos ^{2} \alpha-\frac{\mathrm{E}_{0}}{\mathrm{E}_{90}} \operatorname{sen}^{2} \alpha\right) \cdot \cos (2 \cdot \alpha)+\frac{\mathrm{E}_{0}}{\mathrm{E}_{45}} \operatorname{sen}^{2}(2 \cdot \alpha)}, \operatorname{com} \frac{\mathrm{E}_{0}}{\mathrm{E}_{90}}=7,6 \text { e } \frac{\mathrm{E}_{0}}{\mathrm{E}_{45}}=5,0
$$

Em que:

$\mathrm{E}_{\alpha} \quad=$ módulo de elasticidade na direção inclinada de um ângulo $\alpha$ em relação às fibras da madeira;

$\mathrm{E}_{0} \quad=$ módulo de elasticidade na direção paralela $\left(\alpha=0^{\circ}\right)$ às fibras da madeira;

$\mathrm{E}_{45}=$ módulo de elasticidade na direção inclinada de um ângulo $\alpha=45^{\circ}$ em relação às fibras da madeira;

$\mathrm{E}_{90}=$ módulo de elasticidade na direção normal $\left(\alpha=90^{\circ}\right)$ às fibras da madeira;

$\alpha=$ ângulo entre o esforço aplicado e a direção das fibras; $\frac{\mathrm{E}_{0}}{\mathrm{E}_{90}}=7,6=\begin{aligned} & \text { relação média, obtida para a madeira de peroba-mica, Aspidosperma populifolium A. DC., } \\ & \text { entre os módulos de elasticidade nas direções paralela e normal às fibras; }\end{aligned}$

$\frac{\mathrm{E}_{0}}{\mathrm{E}_{45}}=5,0=$ relação média, obtida para a madeira de peroba-mica, Aspidosperma populifolium A. DC., entre os módulos de elasticidade nas direções paralela e inclinada de $45^{\circ}$ em relação às fibras. 
Aplicando o teste de pairing e o teste dos desvios, na comparação dos resultados dos ensaios (Tabela 1) aos calculados pelo modelo apresentado na equação 12, verificou-se que o intervalo de confiança da média dos desvios, obtidos em cada prancha, ou continha o zero, revelando estimativa justa dos resultados obtidos no modelo, ou apresentava o limite superior também negativo, embora próximo de zero, revelando que o modelo subestimava ligeiramente os módulos de elasticidade, o que, do ponto de vista de segurança das estruturas, é a favor da segurança. O modelo pode ser considerado bom.

Para se avaliar a aplicação dos modelos na rigidez efetiva, para cada inclinação, foi obtida a média (equação 10) dos valores de módulos de elasticidade, apresentados na tabela 1. Esses valores são apresentados na tabela 2 .

Tabela 2. Valores médios do módulo de elasticidade (rigidez efetiva) em cada inclinação.

Table 2. Average values of the module of elasticity (effective stiffness) in each inclination.

Valores médios do módulo de elasticidade, $\mathrm{E}_{\alpha, \mathrm{m}}(\mathrm{MPa})$, para as seguintes inclinações, $\alpha$ :

\begin{tabular}{cccccccccccc} 
& $\mathbf{0}^{\mathbf{o}}$ & $\mathbf{1 0}^{\mathbf{0}}$ & $\mathbf{2 0}^{\mathbf{o}}$ & $\mathbf{3 0}^{\mathbf{0}}$ & $\mathbf{4 0}^{\mathbf{o}}$ & $\mathbf{4 5}^{\mathbf{0}}$ & $\mathbf{5 0}^{\mathbf{0}}$ & $\mathbf{6 0}^{\mathbf{}}$ & $\mathbf{7 0}^{\mathbf{0}}$ & $\mathbf{8 0}^{\mathbf{0}}$ & $\mathbf{9 0}^{\mathbf{0}}$ \\
\hline $\mathrm{E}_{\alpha, \mathrm{m}}(\mathrm{MPa})$ & 13620 & 11033 & 6323 & 4191 & 3061 & 2731 & 2506 & 2132 & 1925 & 1860 & 1855 \\
\hline
\end{tabular}

Aplicando o teste de pairing e o teste dos desvios, na comparação dos resultados obtidos na tabela 2 aos dos modelos apresentados nas equações 1 a 4, foram obtidos, para o módulo de elasticidade médio (rigidez efetiva), os limites do intervalo do expoente " $\mathrm{n}$ " com validade estatística, bem como o valor do expoente "n" que produz o melhor ajuste de cada modelo. Esses resultados, para a madeira de peroba-mica, Aspidosperma populifolium A. DC., indicam:

- A validade da Expressão de Hankinson (equação 1), desde que o expoente "n" esteja no intervalo $1,12 \leq n \leq 1,81$. O expoente que fornece melhor ajuste é $n=1,67$. $O$ valor tradicionalmente utilizado, de $\mathrm{n}=2$, não tem validade estatística.

- A validade da Expressão dos Senos (equação 3), desde que o expoente "n" esteja no intervalo $0,26 \leq n \leq 0,61$. O expoente que fornece melhor ajuste é $n=0,41$. O valor tradicionalmente utilizado pela norma alemã DIN-1052, segundo Pfeil (1978), de $n=1$, não tem validade estatística. Esse modelo também não tem validade com $n=2$ da proposta de Szücs (1992).

- A validade da Expressão apresentada por Karlsen (equação 2), desde que o expoente "n" esteja no intervalo $1,22 \leq n \leq 1,78$. O expoente que fornece melhor ajuste é $n=1,61$. O valor tradicionalmente utilizado, recomendado por Karlsen et al. (1967), de $\mathrm{n}=3$, não tem validade estatística.

- A Expressão de Keylwerth (equação 4) tem validade estatística para " $n$ " no intervalo $1,12 \leq n \leq 2,39$. O expoente que fornece melhor ajuste é $n=2,03$. O valor tradicionalmente utilizado, recomendado por Kollmann; Côté Jr. (1984), de $\mathrm{n}=2$, também tem validade estatística. Esse foi o único modelo que apresentou validade com o expoente com o qual foi originalmente apresentado.

Também para o módulo de elasticidade médio (rigidez efetiva), resolveu-se avaliar o modelo, com base na Expressão de Keylwerth, apresentado na equação 12.

Aplicando-se o teste de pairing e o teste dos desvios, na comparação dos resultados obtidos para o módulo de elasticidade médio (Tabela 2) aos obtidos pelo modelo apresentado na equação 11, verificou-se que o intervalo de confiança da média dos desvios, obtidos para cada variável avaliada, continha o zero, revelando estimativa justa dos resultados obtidos no modelo também para estimar a rigidez efetiva.

Na figura 2 apresenta-se o ajuste dos modelos avaliados à rigidez efetiva definida na NBR 7190 da ABNT (1997), usando-se o expoente tradicionalmente utilizado. Por essa figura percebe-se que apenas dois modelos são estatisticamente ajustados ao modelo proposto por Keylwerth (equação 4) e à proposta, baseada nesse modelo, apresentada na equação 12 . 


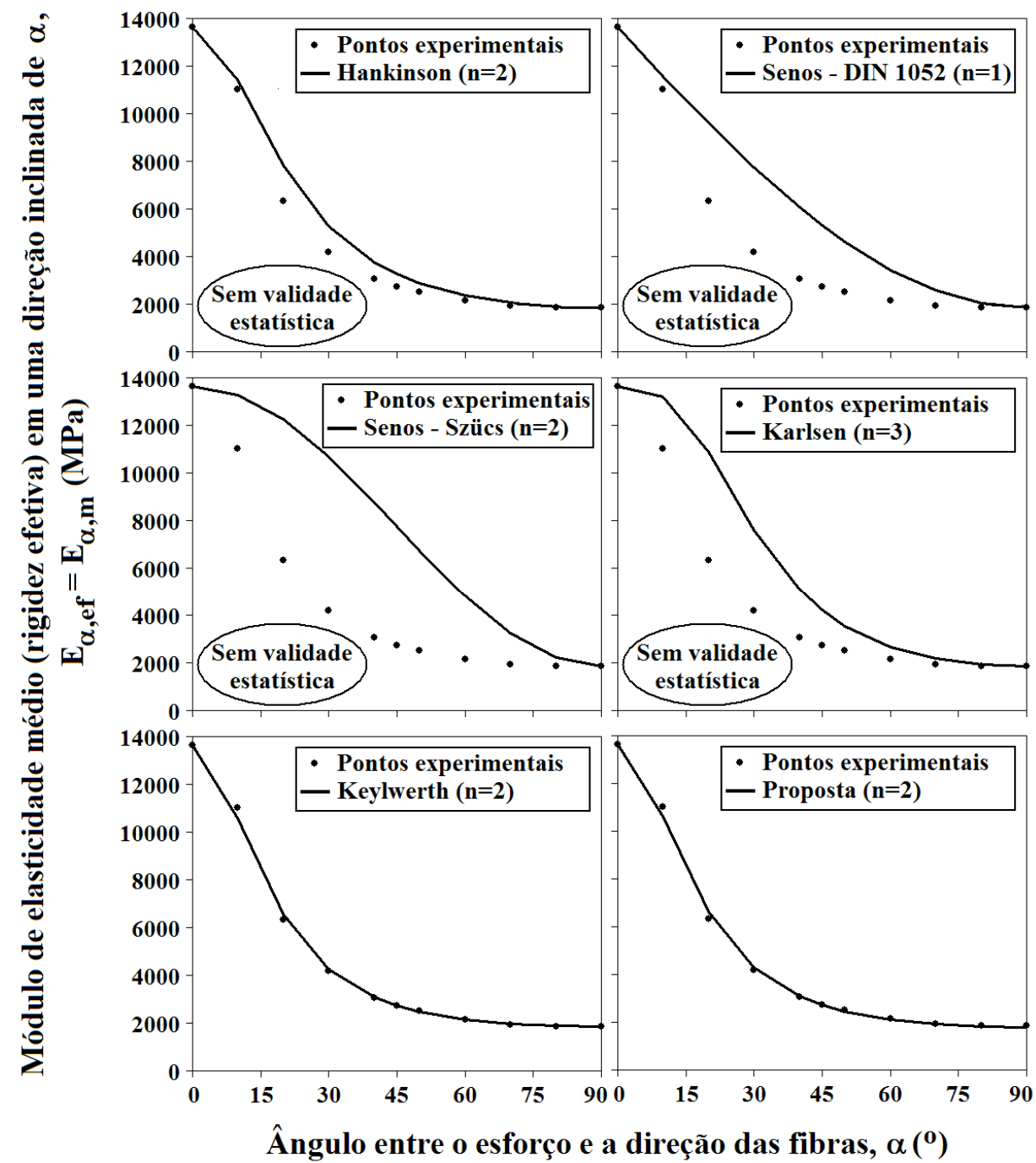

Figura 2. Ajuste dos diversos modelos, com os expoentes tradicionalmente utilizados, aos pontos experimentais correspondentes à rigidez efetiva definida na NBR 7190 da ABNT (1997), ou seja, ao módulo de elasticidade médio.

Figure 2. Adjustment of the several models, with the traditionally used exponents, to the experimental points corresponding to the effective stiffness set in NBR 7190, from ABNT (1997), that is, to the average of the module of elasticity.

Na figura 3 apresenta-se o ajuste dos modelos avaliados à rigidez efetiva definida na NBR 7190 da ABNT (1997), usando-se o melhor expoente para cada modelo. Por essa figura percebe-se que todos os modelos podem ser estatisticamente ajustados, desde que se utilize o expoente adequado. Os modelos empíricos apresentados por Hankinson (equação 1) e por Karlsen (equação 2) forneceram ajustes muito bons, mas os resultados das diversas pranchas indicam que o expoente que fornece melhor ajuste é diferente para cada conjunto de dados experimentais e que pequenas variações no expoente alteram substancialmente o ajuste. 


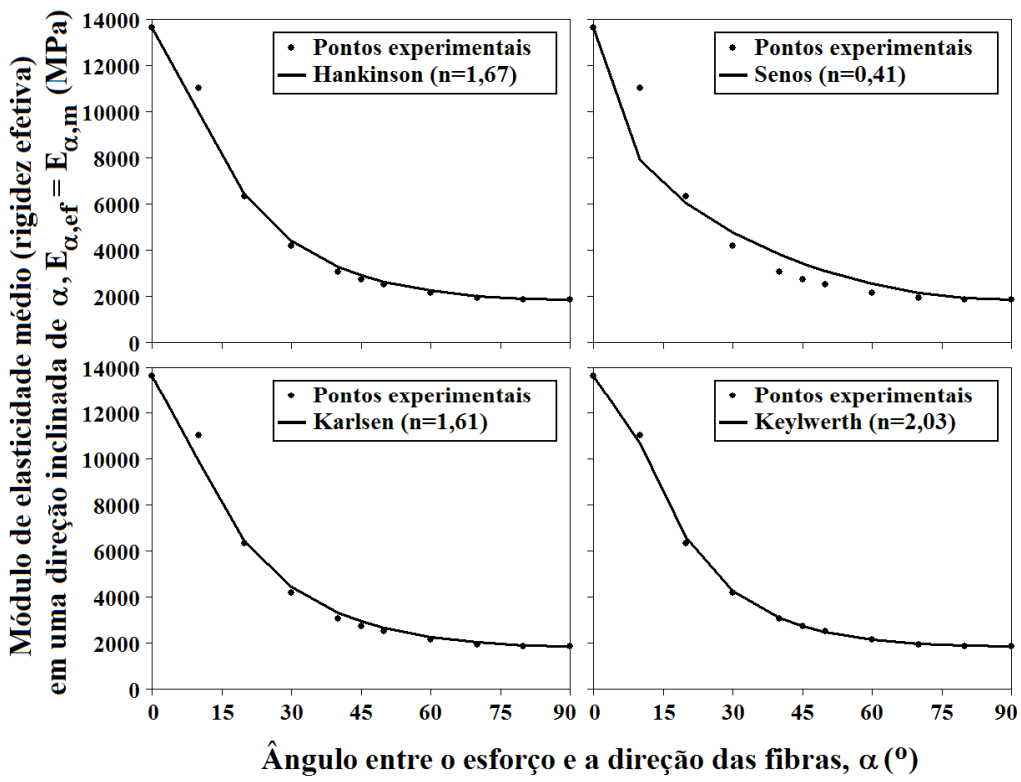

Figura 3. Ajuste dos diversos modelos, com os expoentes que fornecem melhores ajustes, aos pontos experimentais correspondentes à rigidez efetiva definida na NBR 7190 da ABNT (1997), ou seja, ao módulo de elasticidade médio.

Figure 3. Adjustment of the several models, with the exponents that supply the best adjustments to the experimental points corresponding to the effective stiffness set in NBR 7190, from ABNT (1997), that is, to the average of the module of elasticity.

\section{CONCLUSÕES}

Como o trabalho foi realizado com uma única espécie, conhecida vulgarmente por peroba-mica, Aspidosperma populifolium A. DC., as conclusões apresentadas, de maneira generalizada, devem ser entendidas como conclusões para essa espécie, sugestões para reflexão dos revisores da norma brasileira NBR 7190 e recomendações para trabalhos futuros.

- Existe relação funcional entre o módulo de elasticidade e o ângulo entre o esforço aplicado e a direção das fibras da madeira. Dos modelos encontrados na literatura (equações 1 a 4), apenas o modelo de Keylwerth (equação 4) foi considerado estatisticamente válido em seu formato original, tanto para explicar o comportamento individual como o médio (rigidez efetiva). Também se mostrou estatisticamente válido o modelo baseado em Keylwerth utilizando valores fixos para as relações $\mathrm{E}_{0} / \mathrm{E}_{90}$ e $\mathrm{E}_{0} / \mathrm{E}_{45}$. Os valores fixos que foram avaliados correspondem ao valor médio (entre as 12 pranchas) da relação considerada. Para a peroba-mica, Aspidosperma populifolium A. DC., essas relações são as apresentadas, junto com o modelo, na equação 12.

- A utilização da Expressão de Keylwerth (equação 4) é interessante quando for feita a caracterização completa da rigidez da madeira, entretanto será necessário definir em norma o ensaio para a inclinação de $45^{\circ}$.

- Quando for feita caracterização simplificada da rigidez da madeira, pode-se utilizar o modelo baseado em Keylwerth, utilizando valores fixos para as relações $\mathrm{E}_{0} / \mathrm{E}_{90}$ e $\mathrm{E}_{0} / \mathrm{E}_{45}$. Nesse caso, será necessário definir em norma os valores dessas relações.

- A relação " $\mathrm{E}_{\mathrm{c} 90, \mathrm{~m}}=\mathrm{E}_{\mathrm{c} 0, \mathrm{~m}} / 20 \Rightarrow \mathrm{E}_{\mathrm{c} 0, \mathrm{~m}} / \mathrm{E}_{\mathrm{c} 90, \mathrm{~m}}=20$ ", constante da NBR 7190 da ABNT (1997), é diferente da obtida neste trabalho.

- Os outros modelos avaliados, especialmente os de Hankinson (equação 1) e Karlsen (equação 2), com o expoente adequado, também fornecem bom ajuste. Entretanto esses modelos são muito sensíveis à alteração do expoente, portanto obter um expoente estatisticamente aceitável para todas as espécies é tarefa praticamente impossível. 


\section{AGRADECIMENTOS}

Ao Conselho Nacional de Desenvolvimento Científico e Tecnológico (CNPq), pelo financiamento do projeto de pesquisa que deu origem a este trabalho.

\section{REFERÊNCIAS}

ASSOCIAÇÃO BRASILEIRA DE NORMAS TÉCNICAS. NBR 7190 - Projeto de estruturas de madeiras. São Paulo: ABNT. 1997.

DINWOODIE, J. M. Timber its nature and behavior. Princes Risborough Laboratory. Building Research Establishment. New York: Van Nostrand Reinhold Company Ltd., 1991.

HELLMEISTER, J. C. Cálculo de elementos estruturais de madeira à luz da NB-11/51 - Norma Brasileira para o cálculo e a execução de estruturas de madeira. In: ENCONTRO BRASILEIRO EM MADEIRAS E ESTRUTURAS DE MADEIRA, 1, vol. Características, p. 1-51. São Carlos. Anais... São Carlos: USP-EESC-SET-LaMEM, 1983.

KARLSEN, G. G.; BOLSHAKOV, V. V.; KAGAN, M. Y.; SVENTSITSKY, G. V.; ALEKSANDROVSKY, K. V.; BOCHKARYOV, I. V.; FOLOMIN, A. I. Wooden structures. Moscow: Mir Publishers, 1967. 638 p.

KOLlMANN, F. F. P.; CÔTÉ JR., W. A. Principles of wood science and technology. vol. I Solid Wood. New York: Springer-Verlag, 1984.

LAHR, F. A. R. Sobre a determinação de propriedades de elasticidade da madeira. 216 p. Tese (Doutorado em Engenharia de Estruturas) - Escola de Engenharia de São Carlos, Universidade de São Paulo. São Carlos, SP, 1984.

LOGSDON, N. B. Variação da tensão resistente ao cisalhamento paralelo às fibras em uma peça de dimensões comerciais de amescla, Trattinickia burserifolia (Mart.) Wild. In: ENCONTRO BRASILEIRO EM MADEIRAS E ESTRUTURAS DE MADEIRA, 5, v. 2, p. 235-44. Belo Horizonte. Anais... Belo Horizonte: UFMG. 1995.

Influência da umidade nas propriedades de resistência e rigidez da madeira. 174 p. Tese (Doutorado em Engenharia de Estruturas) - Escola de Engenharia de São Carlos, Universidade de São Paulo, São Carlos, SP, 1998.

LOGSDON, N. B.; FINGER, Z.; BORGES, C. G. A. Caracterização físico-mecânica da madeira de peroba-mica, Aspidosperma populifolium A. DC. (APOCYNACEAE). Revista Floresta, v. 38, n. 1, p. 11-21, 2008.

MATEUS, T. J. E. Bases para o dimensionamento de estruturas de madeira. 312 p. Dissertação (Investigador do Laboratório Nacional de Engenharia Civil) - Laboratório Nacional de Engenharia Civil, Ministério das Obras Públicas, Lisboa, 1962.

NICOLAS, E. A.; MASCIA, N. T.; TRINCA, A. J. Determinação da resistência da madeira à tração com ângulo das fibras inclinado em relação ao carregamento. In: ENCONTRO BRASILEIRO EM MADEIRAS E EM ESTRUTURAS DE MADEIRA, 10. São Pedro, SP. Anais... São Carlos: IBRAMEM. 2006. 1 (CD-ROM).

PFEIL, W. Estruturas de madeira: dimensionamento segundo as normas brasileiras NB-11 e os modernos critérios das normas alemãs e americanas. Rio de Janeiro: Livros Técnicos e Científicos, 1978.

SZÜCS, C. A. Estudo do comportamento da madeira a esforços inclinados. In: ENCONTRO BRASILEIRO EM MADEIRAS E EM ESTRUTURAS DE MADEIRA, 4, vol. 2, p. 53-60. São Carlos, SP. Anais... São Carlos: IBRAMEM. 1992. 